\title{
A note against the use of "belonging to" properties \\ in multilevel selection theory
}

\section{Accepted manuscript version. The final publication is available at:}

https://link.springer.com/article/10.1007/s10441-020-09386-9

(Acta Biotheoretica, July 2020)

\author{
Ciprian Jeler \\ Institute for Interdisciplinary Research - Social Sciences and Humanities Research Department, \\ “Alexandru Ioan Cuza" University of Iaşi, Romania
}

\begin{abstract}
In this short paper, I argue against what I call the "belonging to" interpretation of group selection in scenarios in which a group's fitness is defined as the per capita reproductive output of the individuals of the group. According to this interpretation, group selection acts on "belonging to" properties of individuals, i.e. on relational or contextual properties that all the individuals of a group share simply by belonging to that group; thus, if differences in the individuals' "belonging to" properties cause differences in their fitness, group selection sensu the "belonging to" interpretation is said to be at work. I argue that the main problem with the "belonging to" interpretation is that it confuses evolutionary changes due to differences in environmental quality with evolutionary changes due to selection. In other words, I argue that, in the majority of cases, this interpretation actually takes the "selection" out of the "group selection" notion it aims to interpret: by adopting this perspective, one implicitly commits to explaining the evolutionary change under consideration not by a kind of selection (be it individual or group selection), but by differences in the environmental quality experienced by individual types.
\end{abstract}

\section{Introduction}

In this short paper, I argue against a particular interpretation of group selection that seems to be endorsed in a fraction of the multilevel selection literature. In multilevel selection theory, the evolution of certain traits - be they traits of cells, organisms, communities, species etc. - is explained by appealing to the idea that natural selection may simultaneously operate on at least two levels of biological organization. Multilevel selectionist explanations are often used with respect to the evolution of traits known as altruistic (e.g. Sober and Wilson 1998) or with respect to major evolutionary transitions (e.g. the evolution of multicellularity - Michod 1999). When just two levels are considered, the lower-level entities on which selection acts are usually called "individuals", whereas the higher-level entities, within which individuals are nested, are referred to as "groups". Thus, in any two-level selection scenario, "group selection" usually denotes the selection process taking place at the higher level of organization. It is customarily accepted that group selection may be of two kinds, depending on whether we consider a group as fitter than another one if it produces 
more offspring groups (this is usually called "multilevel selection 2" or MLS2) or if it has a higher output of individuals per individual member (this is usually referred to as "multilevel selection 1" or MLS1 ${ }^{1}$; see Damuth and Heisler 1988; Mayo and Gillinsky 1987).

My discussion here only concerns group selection of the latter kind: therefore, in the rest of this paper, the term group selection will refer to the MLS1 type. I will show (Section 2) that there are (at least) two manners in which this kind of group selection is interpreted in the literature: one is a proper "group-level interpretation," while the other one is an individual-level interpretation according to which group selection acts on "belonging to" properties of individuals, where a "belonging to" property is a relational (or contextual) property that is shared by all the individual members of a group simply because they belong to that group. I will then go on to provide (in Section 3) a cascading argument against this "belonging to" interpretation. More precisely, I argue that, in the majority of cases, this interpretation actually takes the "selection" out of the "group selection" notion it aims to interpret: embracing this interpretation boils down to claiming that the evolutionary change under consideration results not from selection (at whatever level that may be), but from differences in the environmental quality encountered by the individual types of the focal population.

Conceptual clarification of the "belonging to" interpretation is the main motivation behind my discussion here. And the conclusion of this clarification is that, by adopting the "belonging to" interpretation, one commits to a position that explains (part of) the focal evolutionary change not by selection (and implicitly not by group selection), but by differences in the environmental quality experienced by individuals.

\section{Two interpretations of group selection}

\subsection{The group-level interpretation}

Imagine we have two groups $(\mathrm{O}$ and $\mathrm{P})$ that vary with respect to a given group-level trait $X$, with group $\mathrm{O}$ having a higher value for $X$ than group $\mathrm{P}$. One way to analyze a case of this sort is to define a group's fitness as the average fitness of the group's individual members and to go on to determine whether the difference in group character between the groups causes a difference in fitness between them. ${ }^{2}$ Thus, if group $\mathrm{O}$ is fitter than group $\mathrm{P}$ due to its higher level of $X$, then our case is one of group selection. This encapsulates the group-level interpretation of group selection.

\footnotetext{
${ }^{1}$ My opinion is that the terms MLS1 and MLS2 should not be used in order to designate these two kinds of group selection and should be reserved for a different usage. However, since these terms are largely used in the literature in this sense, I will also adopt this terminology in this paper.

${ }^{2}$ I adopt here a causal view of selection that is becoming increasingly popular among evolutionary theorists in general (e.g. Sober 1984; Shanahan 1990; Waters 2005; Glymour 2006, 2011; Godfrey-Smith 2007; Otsuka 2016), as well as among authors specifically working on multilevel selection (e.g. Arnold and Fristrup 1982; Okasha 2006; Jeler 2017).
} 
Now suppose that the individuals of the two groups are of two types, where the types are distinguished only by their colour: half of the individuals are red, while the other half are grey. Moreover, suppose that the two groups of this toy example also vary with respect to the proportion of types they contain, with group $\mathrm{O}$ containing more grey individuals than group $\mathrm{P}$. Given that grey individuals are better represented in the fitter group $\mathrm{O}$, they will increase in frequency as an indirect result of group-level selection on $X^{3}$

This verdict may seem somewhat counterintuitive. Indeed, one individual type is said to increase in frequency due to selection on the group-level trait $X$, but this group-level selection is based on a notion of group fitness that is itself mathematically dependent on the fitnesses of individuals. In other words, the verdict states that certain individuals receive a boost in fitness - and therefore in frequency - because of a group-level differential fitness that is itself defined by appealing to individual fitness.

The reply to this worry is that the counterintuitiveness may be allayed if we consider the cases where group selectionist explanations are most compelling. Okasha (2016) has convincingly argued that, in certain cases, group selectionist explanations are preferable to kin selectionist ones because the former kind of explanation captures the direct causal influences between the salient variables, whereas the latter kind of explanation - though not flawed - uses variables that are only indirectly causally connected. The specific cases Okasha has in mind are those in which it is the group "that has a fitness value in the first instance," whereas the individual fitness is only "derivative" (Okasha 2016, 455); the most straightforward examples - but probably not the only ones - are cases in which the groups in question exhibit reproductive division of labor (this is, for example, the case of eusocial insects where only a minority of organisms does the reproducing for the entire colony, or of multicellular organisms in which somatic cells have no access to the germ line etc.). In this kind of cases, a given group-level trait may very well fully determine the reproductive success of the whole group ${ }^{4}$, and the per capita reproductive output of the individuals of the group - e.g. the average number of copies left by the alleles at a given locus - only depends on how the total reproductive output of the group is shared among its members. In such cases, claiming that a given individual-level trait may spread because of selection on a group-level trait is no longer counterintuitive. But let me also emphasize that the issue of how to interpret the notion of group selection and the issue of when group selectionist explanations are preferable to other kinds of explanation are two theoretically decoupled issues and my interest in this paper lies with the former, rather than the latter.

\footnotetext{
${ }^{3}$ Throughout this paper, I assume that individuals faithfully transmit their type to their offspring.

${ }^{4}$ The trait may be, for example, the degree of reproductive competition in certain ant species, where an overabundance of egg-laying workers leads to a lack of workers taking care of the brood (Hölldobler and Wilson 2009).
} 
The main characteristics of the group-level interpretation may thus be summarized as follows:

a) it sees "group selection" as acting on a group-level property. The groups themselves are under selection here, even though a group's fitness is definitionally dependent on the fitnesses of its individual members;

b) individual types change in frequency as a result of selection at the higher level. There is, therefore, a presumed "vertical" effect of group selection on the frequencies of individual types.

Let us briefly add that the list of publications endorsing the "group-level interpretation" of group selection includes: Wilson (1975, 1989), Sober (1984), Sober and Wilson (1998), Michod (1999).

\subsection{The "belonging to" interpretation}

But there is a second way in which the above toy scenario could be interpreted. A few steps need to be taken for this:

i) First, we assign a relational or a "belonging to" property to each individual of our case, depending on the value for $X$ of the group it belongs to. More precisely, all the individuals in group $\mathrm{O}$ will have the same value for this "belonging to" variable and this value will be equal to $X_{O}$, i.e. to the level of $X$ of group O. Similarly, the value of this "belonging to" variable for each individual in group $\mathrm{P}$ will be equal to $X_{P}$.

ii) We then determine whether having a "belonging to" property whose value is equal to $X_{O}$ increases an individual's fitness with respect to other individuals in the population (in our case, with respect to individuals with an $X_{P}$ value for their "belonging to" property).

iii) If it does, then we have "group selection" here sensu the "belonging to" interpretation.

Furthermore, we can now note that there is a correlation in our population between individual color and the value for the "belonging to" variable: most of the grey individuals of the population have a "belonging to" variable equal to $X_{O}$, whereas most of the red individuals have a lower value for their "belonging to" variable, namely $X_{P}{ }^{5}$ As a consequence, because there is a correlation between "greyness" and higher value for the "belonging to" variable, grey individuals will increase in frequency in the global population as an indirect result of selection on the "belonging to" properties of individuals.

To sum up, the main characteristics of the "belonging to" interpretation are the following:

\footnotetext{
${ }^{5}$ Whether this correlation is spurious or not will only become relevant later on.
} 
a) it sees "group selection" as acting on an individual-level property. It is the individuals that are under selection here, even though the manner in which we assign a value for the "belonging to" property of individuals is dependent on the relevant group-level property;

b) individual types change in frequency as a result of selection at their own (individual) level. There is, therefore, a presumed "horizontal" effect of group selection on the frequencies of individual types, just like in any case in which two associated organism-level traits spread together even when selection is only acting on one of them.

Before we go on to criticize this "belonging to" interpretation, we should first ask ourselves whether there are authors who do endorse it. What seems to be the most clear-cut endorsement of this interpretation is found in the seminal paper that has put forth the distinction between multilevel selection of the MLS1 and MLS2 kinds, namely Damuth and Heisler (1988). Here is how they present MLS1:

In multilevel selection [1]:

(1) "Group selection" refers to the effects of group membership on individual fitness.

(2) Fitnesses are properties of individuals.

(3) Characters are values attributed to individuals (including both individual and contextual characters) (Damuth and Heisler 1988, 410).

The crucial elements of the "belonging to" interpretation are all here. Damuth and Heisler stress that it is to the individuals that the characters relevant for selection are attributed, and this includes "contextual" characters. A few pages later they clarify what they mean by such characters by saying that "any character of the group to which an individual belongs, including group means or frequencies, can be formally assigned to that individual as a result of its membership in the group; such a character becomes a contextual character of that individual, and its value is identical for all members of the group" (ibid., 417-418). Thus, contextual characters are attributed to individuals, their value is dependent on the value of the group characters and is thus identical for all the individuals of the group: these are exactly our "belonging to" properties. Moreover, Damuth and Heisler also stress that the relevant fitnesses are properties of individuals. In other words, selection here refers to differences in individual fitness that are due to differences in values of the "belonging to" properties of individuals, or, as they put it, group selection of the MLS1 kind "refers to the effects of group membership on individual fitness." Whether or not we should read the quoted fragment from Damuth and Heisler as an attempt to outline a particular interpretation of group selection is unclear. But, if we do read it in this way, it would be difficult to find a clearer endorsement of the "belonging to" interpretation in the literature than the one from their highly influential article. ${ }^{6}$

\footnotetext{
${ }^{6}$ There are, however, some phrases in this article that seem to gesture towards an endorsement of the "group-level interpretation." For example, when they speak of group selection of the MLS2 kind, Heisler and Damuth $(1988,410)$
} 
A less clear-cut endorsement of the "belonging to" interpretation is found in Evolution and the Levels of Selection, Samir Okasha's 2006 book that has set the tone for philosophical discussions of multilevel selection in recent years. Precisely because it is less clear-cut, discussing this case requires some background information and more extensive quoting. First, let us recall that, in a parallel paper to the one quoted above, Heisler and Damuth (1987) had proposed "contextual analysis" as a statistical method for analyzing multilevel selection scenarios of the MLS1 kind. This method consisted in extending the multiple regression technique for the analysis of selection on multiple correlated characters developed by Lande and Arnold (1983) so that it could also accommodate contextual characters, i.e. relational characters that the individuals posses simply by belonging to a given group (in our terms: "belonging to" characters). As Heisler and Damuth (1987, 588) state, in contextual analysis, contextual characters "are quantifiable group properties treated as individual traits. Thus, a contextual character is a component of an individual's phenotype that is shared identically by all members of a particular group." It is therefore highly likely that Damuth and Heisler's (1988) apparent endorsement of the "belonging to" stems from their preference for contextual analysis as a method for analyzing multilevel selection scenarios (more on this below). ${ }^{7}$

Okasha (2006) picks up where Heisler and Damuth (1987) left off. His discussion starts by unambiguously stating that one of the essential features of MLS1 is that "collective [= group] fitness is defined as average particle [= individual] fitness" (Okasha 2006, 85); similarly, he states that Wislon's (1975) trait-group model for the evolution of altruism is an example of MLS1 scenario (Okasha 2006, 56) in that it "treats groups as fitness-bearing entities" (57). Moreover, just like I do here, he endorses a strictly causal view of natural selection according to which we are dealing with selection when a character "causally influences fitness," which is more precisely expressed by saying that "differences in the character cause fitness differences" (Okasha 2006, 76). By putting these two elements together, we get a picture that is fairly similar to the group-level

state: "In this case, we have identified a different kind of fitness than in the first [i.e. than in the MLS1 case], a grouplevel fitness that is not simply the mean of the fitnesses of the group's members," which suggests that the relevant fitness for MLS1 is the mean fitness of a group, as in the "group-level interpretation" presented above.

${ }^{7}$ Without dwelling on its details here, let me recall that, in MLS1 scenarios, contextual analysis allows us to partition the population average change in an individual-level character $z$ in the following manner:

$\Delta \bar{z}=\frac{\beta_{z} \operatorname{Var}\left(z_{i j}\right)}{\bar{w}}+\frac{\beta_{Y} \operatorname{Cov}\left(z_{i j}, Y_{j}\right)}{\bar{w}}$, where $z_{i j}$ is the character of the $i^{\text {th }}$ individual in the $j^{\text {th }}$ group, $Y_{j}$ is the group character of the $j^{\text {th }}$ group, $\bar{w}$ is the average individual fitness in the global population, $\beta_{z}$ is the partial regression coefficient of individual fitness on individual character and $\beta_{Y}$ is the partial regression coefficient of individual fitness on group character. In cases in which the relevant group character is defined as the average character of its individual members, the equation becomes $\Delta \bar{z}=\frac{\beta_{z} \operatorname{Var}\left(z_{i j}\right)}{\bar{w}}+\frac{\beta_{Z} \operatorname{Cov}\left(z_{i j}, Z_{j}\right)}{\bar{w}}$, where $Z_{j}$ is the average individual character in the $j^{\text {th }}$ group. In the latter kind of cases, a different partition is provided by Price's (1972) hierarchical equation, namely: $\Delta \bar{z}=\frac{E\left[\operatorname{Cov}\left(z_{i j}, w_{i j}\right)\right]}{\bar{w}}+\frac{\operatorname{Cov}\left(Z_{j}, W_{j}\right)}{\bar{w}}$, where $w_{i j}$ is the fitness of the $i^{\text {th }}$ individual in the $j^{\text {th }}$ group and $W_{j}$ is the average fitness of the individuals of the $j^{\text {th }}$ group. Heisler and Damuth (1987) argue for the superiority of the contextual analysis partition over that of Price's equation. 
interpretation discussed above: if a difference in group character causes a difference in group fitness (defined in the MLS1 way, as average fitness of the groups' members), then we have group selection. So when Okasha moves on and discusses the discrepancies between Price's equation and contextual analysis, his discussion should try to determine which one is better at isolating the effects of group selection on the change in the focal individual-level trait: in Okasha's $(2006,94)$ terms, which of the two equations indicated above provides a more accurate "causal decomposition" of the evolutionary change.

However, as his discussion progresses, and for reasons that I will not dwell on here ${ }^{8}$, Okasha is lead to argue that Price's equation and contextual analysis are not merely two ways of partitioning the evolutionary change, but that they represent two different "approaches" to MLS1 or, in his own terms, "they embody conflicting conceptions of multi-level selection" (Okasha 2006, 94). This blurs the distinction between contextual analysis as a mere instrument for measuring the effects of group selection (however the latter may be construed) and the contextual "approach" as a conception regarding what group selection is. ${ }^{9}$ So when, for example, following Heisler and Damuth (1987), Okasha $(2006,86)$ states that "The basic idea [of contextual analysis] is to regard a collective's character as a 'contextual' character of each particle in the collective," it is no longer certain whether this is seen just as a formal convention useful for purposes of measuring the effects of group selection or whether this is taken to operate a substantial modification of the notion itself of group selection. Moreover, towards the end of his discussion on this, Okasha $(2006,93)$ does state that "contextual analysis is intrinsically reductionistic" in that it "treats the collectives [= the groups] as part of their [the individuals'] fitness-affecting environments." This is precisely the perspective assumed by the "belonging to" interpretation: the groups are not themselves under selection, they just provide the different environments or contexts over which an essentially individual-level type of selection is operating.

To sum up, I would say that it is unclear whether Okasha (2006) does indeed endorse the "belonging to" interpretation, but there are enough elements that might lead readers into thinking that such an endorsement is found therein.

Another apparent endorsement of the "belonging to" interpretation is found in Glymour (2008), the article from which I have borrowed the term "belonging to" property. Glymour introduces it by saying that "belonging to" properties are "surrogates for distinct but definitionally related 'group-level' variables; because these surrogates are defined for individuals, they can be

\footnotetext{
${ }^{8}$ One of these reasons is certainly the fact that "contextual analysis" detects a group selection component in cases of "soft selection," i.e. cases in which, overall, groups do not vary in fitness, yet the fitnesses of individuals are influenced by their group's character. For some recent attempts to solve this thorny issue, see Goodnight (2013) and Bourrat (2016).

${ }^{9}$ Indeed, I suspect that Damuth and Heisler's (1988) failure to acknowledge this distinction has occasioned their abovedetailed apparent endorsement of the "belonging to" interpretation.
} 
used in a causal or a statistical analysis in which the individual is the unit of analysis" (Glymour 2008, 836). These surrogate "belonging to" properties could thus be seen as convenient notational instruments for tracking the effects of group selection on the frequencies or average values of individual-level traits. In any case, using such surrogate "belonging to" properties does not seem to necessarily commit one to an interpretation about whether group selection acts on a group-level property or whether it acts on "belonging to" properties of individuals. However, as Glymour's discussion goes on, he does seem to commit to the latter position and he ends up explicitly stating that "group selection acts whenever belonging to properties causally influence reproductive success" (Glymour 2008, 844). Though not lacking in ambiguity, I take Glymour's discussion to constitute a fairly clear endorsement of the "belonging to" interpretation. But, interestingly, Glymour is very critical of contextual analysis and this shows that one can endorse the "belonging to" interpretation without also endorsing contextual analysis as a method for analyzing group selection.

\section{A cascading argument against the "belonging to" interpretation}

Before we ask whether group selection may be said to act on "belonging to" properties of individuals, we should first ask whether natural selection in general - at whatever level - may act on properties of this sort. The answer to this latter question has to be negative: seeing natural selection in general as acting on "belonging to" properties would lead us to confuse evolutionary changes due to differences in environment with evolutionary consequences of selection.

To see this, let us look at an example similar to one from Brandon (1990). Imagine a population of organisms spread over two environments that vary in quality (one is good for the organisms in question, the other one is poor). In a case of this sort, belonging to the better environment does boost one's fitness with respect to organisms belonging to the poor environment: we would thus have to conclude that there is selection on the "belonging to" properties of organisms here. So, instead of drawing the intuitive conclusion that any evolutionary consequences that may arise here are simply due to differences in the environments encountered, by appealing to "belonging to" properties we would have to claim that these consequences stem from selection. In Brandon's $(1990,64)$ terms, we thus confuse "survival of the fittest" with "survival of the luckiest."

This example is not a singular one. Indeed, if we were to embrace the idea that selection may act on "belonging to" properties of biological entities, we would be hard pressed to find any subsisting example of evolution due to chance. Even the classical example of lightning strikes would turn into a selection example: "belonging to" the set of organisms struck by lightning bolts does cause one's fitness to drop, therefore selection on this "belonging to" property is acting; the evolutionary changes due to lightning strikes would thus become consequences of selection. Any 
obvious example of evolution due to chance becomes a case of natural selection once we adopt the idea that selection may act on "belonging to" properties: I presume this is something most philosophers and - probably to a greater extent - most biologists would want to avoid.

Someone could agree that natural selection in general should not be seen as acting on "belonging to" properties, but still contend that such properties may nonetheless be used in multilevel selection theory, and more specifically in cases in which individuals possessing such traits "belong to" genuine groups (exactly how such groups should be identified and demarcated is an issue I will not tackle here ${ }^{10}$ ). In order to asses this possibility, let us begin by looking at a hypothetical toy example in which two groups (A and B) vary with respect to a given group-level property like density ${ }^{11}$ and the average individual fitness in group A is higher due to the group's higher density; let us further suppose, for the time being, that a group's density does not depend in any way on the traits of its individual members.

Looking at this toy case from the perspective of the group-level interpretation, we may say that if group A outreproduces group B because of its higher density, then group-level selection is operating. But when we look at the case from an individual perspective - as the "belonging to" interpretation demands us to -, we have to say that the individuals in group A just happen to encounter living conditions that include a higher density, and they have, on average, a higher fitness as a consequence. We thus admit that some individuals of the global population happen to be distributed in the better environment, whereas the others are distributed in the poorer environment. There is no substantial difference between this case and the Brandon-like case discussed above: claiming that we are dealing with selection here because differences in "belonging to" properties of individuals cause differences in fitness between them is just as problematic here as it was there. Thus, claiming that, in this case, selection acts on "belonging to" properties again comes down to confusing "survival of the fittest" with "survival of the luckiest".

To put it otherwise, this sort of case may be seen as a case of "selection" only when we look at it from a group-level perspective (and we thus take a difference in a group-level trait - density to cause a difference in group fitness between the two groups). When we look at the exact same case from the individuals' perspective, it becomes a case of evolution due to differences in environment: some individuals encounter better living conditions than others and this in no way depends on the traits of these individuals. It follows that one may still adopt an individual-level perspective with respect to this case: however, if one does adopt it, one should not claim that the evolutionary change in this case is a consequence of selection of any sort (be it "group" or

\footnotetext{
${ }^{10}$ For detailed treatments of this issue, see Sober (1984), Okasha (2006), Godfrey-Smith (2008) and Glymour (2017).

${ }^{11}$ I use density as the group character of interest because, as Damuth and Heisler (1988) note, this is a property that cannot be measured at the level of individuals.
} 
"individual" selection); rather, one should state that it is just a consequence of the uneven distribution of types into environments of different quality.

One might object that in many cases individuals do not just happen to belong to the group they are in: often there are biological reasons for their belonging to one group or another. This is correct and it will help us distinguish more clearly between cases of evolution due to differences in environment and cases involving selection. To see this, let us compare the following two versions of our case:

a) First, let us suppose that group density is stable across multiple generations and that there is a reason why the individuals of the higher-density group A are in that group, namely they were born in it. But note that, in this modified case, the important factors remain as in the previous one: an individual in group A experiences a more favorable social environment (i.e. a higher group density) than an individual in group B, but this difference in social environment is in no way due to a difference in these individuals' traits. Therefore, saying that there is selection on "belonging to" properties here is tantamount to saying that there is selection for the relevant living conditions encountered by the individuals (here, selection for the density encountered). Therefore, even in cases in which there is a reason (e.g. ancestry) why individuals are found in the groups they belong to, adopting a "belonging to" interpretation with respect to such a case would again lead us to take evolutionary changes due to differences in environmental quality to be evolutionary consequences of selection.

b) Now let us suppose that the individuals that end up in group A do so because they prefer groups with a higher density. Their belonging to that group thus occurs not by chance, nor by birth, but by choice. In this modified case, saying that selection acts on the "belonging to" properties of the individuals seems legitimate, given that the difference in the individuals" "belonging to" properties is a result of a difference in their density preferences or, more generally, in their preferences for particular social environments. But this also means that here the "belonging to" properties of individuals are caused by - and their values simply reflect - these individuals' social environment preferences. However, these preferences are prima facie much more entitled to be considered phenotypic traits than are "belonging to" properties, mainly because the former are intrinsic properties of individuals, whereas "belonging to" properties are strictly relational ones (indeed, there are authors who claim that only intrinsic properties of biological entities may be considered phenotypic traits on which natural selection acts - e.g. Godfrey-Smith 2009). Therefore, in this sort of case, instead of appealing to "belonging to" properties, it is much more straightforward to say that selection acts on the preferences of individuals for certain social environments (Brandon 1990). 
The take-away point from this discussion is that we dealing with "evolution due to differences in environment" when the difference in living conditions (here, in social environment or density) that the individuals encounter is not due to a difference in trait between these individuals. On the other hand, we are dealing with selection when the difference in the living conditions encountered is due to a difference in individual trait (here, in density preferences); however, in such cases, it is much more straightforward to claim that selection acts on that individual trait (i.e. on density preferences), rather than on the "belonging to" properties that derive from it.

One might agree with this argument, but claim that it only narrows down the applicability range of the "belonging to" interpretation: if the latter is problematic when there is no dependency relation between the relevant group trait and the individual traits of the group's members, then it might well work when such dependencies (be they causal or definitional) are in place.

But this last refuge does not succeed in saving the "belonging to" interpretation either. To see this, let us again imagine that two groups differ with respect to their value for a trait $Y$ that is causally or definitionally dependent on the trait $y$ of the individuals that make up the groups. Therefore, a group has a high value for $Y$ because it mostly contains individuals possessing trait $y$. Here, one may argue that an individual that belongs to the group with a higher $Y$ will benefit from the better living conditions ensured by the higher $Y$ not by accident, but because it will have itself contributed to the bringing about of these better living conditions (i.e. of the higher $Y$ ). The problem with this argument, though, is that it fails to notice that that individual's trait is only responsible for a fraction of the living conditions that that individual encounters, while most of these living conditions depend on the traits of the other members of the individual's group. If, again, we compare two individuals from the two groups, we will have to conclude that a fraction of the difference in living conditions they encounter may be due to a difference in trait $y$ between the two individuals, but that most of the difference between their living conditions is due to the difference in the social environment they encounter. So, just like with respect to density above, we must inquire about the source of this difference in social environment: is it due to a difference in trait between the two individuals or not? And, depending on the answer to this question, we again have two possibilities:

a) If the difference in social environment is not due to a difference in trait between individuals, saying that there is selection on "belonging to" properties here is tantamount to saying that there is selection for the living conditions encountered by the individuals. This, however, is just as problematic as in the cases discussed above. It is important to stress that this point holds even for what is probably the most common and the most relevant kind of case for the evolution of social behavior, namely the type of case in which certain factors (e.g. limited dispersal) ensure that groups are mainly composed of genetically related individuals. In such cases, again, there is an underlying 
reason for the correlation between individual trait $y$ and the group trait $Y$ (i.e. for the fact that most individuals possessing $y$ are clumped into one group and most individuals that do not posses $y$ are found in the other group), and that reason is the common ancestry of the members of each group. But, as discussed above with respect to the density case, this is not sufficient for legitimizing the idea that there is selection on "belonging to" properties: it is not sufficient that there be $a$ cause for the difference in the social environment encountered by two individuals from different groups; what is required is that this difference itself be the result of a difference in trait between these two individuals. And, because this requirement is not met here, claiming that there is selection for "belonging to" properties in cases with limited dispersal is, again, tantamount to claiming that there is selection for the quality of the environment encountered by the individuals. ${ }^{12}$

b) On the other hand, the difference in the social environment experienced might be due to a difference in trait between individuals if, for example, individuals of one type prefer to assort with their own kind. Here, just like in version b) of the density case above, most individuals possessing $y$ are found in the same group (that consequently has a higher $Y$ ) not by chance, nor by birth, but by preference. But this assortative preference is, of course, just another form of preference for a particular social environment and what I said above about the latter holds here as well: selection may indeed be said to act on "belonging to" properties here, but it is much more straightforward to claim that selection acts on the assortative preferences themselves, rather than on the "belonging to" properties that derive from them.

To sum up, claiming that there is selection on "belonging to" properties seems legitimate only in cases in which the difference in the social environment encountered by individuals is due to a difference in trait between these individuals, as is the case, for example, when individuals exhibit preferences for certain kinds of social environment; however, in these cases, it makes more sense to

\footnotetext{
${ }^{12}$ It is crucial to stress that the kind of argument I am making here against the "belonging to" interpretation in populations with limited dispersal does not apply to kin selection theory. Like the "belonging to" interpretation, kin selection approaches do distinguish between two components of individual fitness, namely direct fitness, on one side, and, on the other side, indirect fitness (in the inclusive fitness approach) or the fitness benefit brought to the focal individual by genetically similar individuals (in the neighbor-modulated fitness approach). However, and this is the crucial difference, unlike the "belonging to" interpretation, kin selection approaches do not claim that the two components of fitness correspond to two different selection processes (namely, individual selection on the relevant individual trait, on one side, and selection on "belonging to" properties, one the other). On the contrary, when one says that a particular trait/behavior spreads by kin selection, this means that the combined effects of the two components of fitness lead to an increase in frequency of that trait/behavior. To put it otherwise, even though one may separate the components of fitness of each individual at a given moment (e.g. into direct and indirect fitness, in the inclusive fitness approach), it is the sum of these components that is taken as the fitness notion corresponding to the process of (kin) selection for the focal trait/behavior of that individual. It is this extension of the notion of fitness that constitutes the distinguishing feature of kin selection theory (see Marshall 2015). Indeed, Hamilton $(1964,8)$ is explicit about this: "Just as in the sense of classical selection we may consider whether a given character expressed in an individual is adaptive in the sense of being in the interest of his personal fitness or not, so in the present sense of selection we may consider whether the character or trait of behaviour is or is not adaptive in the sense of being in the interest of his inclusive fitness."
} 
claim that selection acts precisely on these preferences of individuals, rather than on the "belonging to" properties deriving from them.

In contrast, note that the group-level interpretation has no problem accommodating the above-discussed toy cases ${ }^{13}$. If two groups differ in fitness due to a given group-level trait (that may or may not depend on the traits of the groups' members), then group selection sensu the group-level interpretation may be said to act, and this irrespective of whether the uneven distribution of individual types into groups is accidental or is due to descent or social environment preferences.

\section{Conclusion}

In this short paper, I argued against the "belonging to" interpretation of MLS1 group selection that is endorsed - or, at least, seems to be endorsed - in a fraction of the multilevel selection literature. I argued that, in a minority of cases (when the individuals' belonging to their group is due to their preferences for certain social environments), this interpretation might be more sensibly replaced with the idea of selection for these preferences of individuals. However, in the majority of cases, the "belonging to" interpretation takes the "selection" out of the "group selection" notion it intends to interpret: by adopting this perspective with respect to a given scenario, one implicitly commits to explaining the evolutionary change therein not by a kind of selection (be it individual or group selection), but by differences in the environmental quality experienced by individual types.

On the other hand, I pointed out that the arguments given here against the "belonging to" interpretation do not affect the group-level interpretation of group selection. This suggests that, if we want multilevel selection theory to be a theory about selection, endorsing the group-level interpretation of group selection is a suitable option.

\section{Acknowledgements}

I would like to thank two anonymous reviewers for their comments on a draft of this paper. This work was supported by a project funded by the Romanian Ministry of Research and Innovation within Program 1 - Development of the national RD system, Subprogram 1.2 - Institutional Performance - RDI excellence funding projects, Contract no.34PFE/19.10.2018.

\section{References}

Arnold AJ, Fristrup K (1982) The theory of evolution by natural selection: A hierarchical expansion. Paleobiology 8:113-129

Bourrat P (2016) Generalizing contextual analysis. Acta Biotheor 64:197-217

Brandon RN (1990) Adaptation and environment. Princeton University Press, Princeton

\footnotetext{
${ }^{13}$ And, as pointed out above, nor does kin selection theory.
} 
Damuth J, Heisler L (1988) Alternative Formulations of Multilevel Selection. Biol Philos 3:407430

Glymour B (2006) Wayward modeling: population genetics and natural selection. Philos Sci 73:369-389

Glymour B (2008) Correlated interaction and group selection. Br J Philos Sci 59:835-855

Glymour B (2011) Modeling environments: interactive causation and adaptations to environmental conditions. Philos Sci 78:448-471

Glymour B (2017) Cross-unit causation and the identity of groups. Philos Sci 84:717-736

Godfrey-Smith P (2007) Conditions for evolution by natural selection. J Philos 104:489-516

Godfrey-Smith P (2008) Varieties of population structure and the levels of selection. Br J Philos Sci 59: $25-50$

Godfrey-Smith P (2009) Darwinian populations and natural selection. Oxford University Press, Oxford

Goodnight CJ (2013) On multilevel selection and kin selection: contextual analysis meets direct fitness. Evolution 67:1539-1548

Hamilton WD (1964) The genetical evolution of social behaviour, I. J Theor Biol 7:1-16

Heisler IL, Damuth J (1987) A method for analyzing selection in hierarchically structured populations. Am Nat 130:582-602

Hölldobler B, Wilson EO (2009) The superorganism: The beauty, elegance, and strangeness of insect societies. W. W. Norton, London

Jeler C (2017) Multi-level selection and the issue of environmental homogeneity. Biol Philos 32:651-681

Lande R, Arnold SJ (1983) The measurement of selection on correlated characters. Evolution $37: 1210-1226$

Marshall, JAR (2015) Social evolution and inclusive fitness theory: An introduction. Princeton University Press, Princeton

Mayo DG, Gilinsky NL (1987) Models of group selection. Philos Sci 54:515-538

Michod RE (1999) Darwinian dynamics: Evolutionary transitions in fitness and individuality. Princeton University Press, Princeton

Okasha S (2006) Evolution and the levels of selection. Oxford University Press, Oxford

Okasha S (2016) The relation between kin and multilevel selection: An approach using causal graphs. Br J Philos Sci 67:435-470

Otsuka J (2016) A critical review of the statisticalist debate. Biol Philos 31:459-482

Price G (1972) Extension of covariance selection mathematics. Ann Hum Gen 35:485-490 
Shanahan T (1990) Evolution, phenotypic selection and the units of selection. Philos Sci 57:210 225

Sober E, Wilson DS (1998) Unto others: the evolution and psychology of unselfish behavior. Harvard University Press, Cambridge

Sober E (1984) The nature of selection: Evolutionary theory in philosophical focus. MIT Press, Cambridge

Waters CK (2005) Why genic and multilevel selection theories are here to stay. Philos Sci 72: 311333.

Wilson DS (1975) A theory of group selection. PNAS 72:143-146

Wilson DS (1989) Levels of selection: an alternative to individualism in biology and the human sciences. Soc Netw 11: 257-272 